\title{
Induction of GNE in Myofibers after Muscle Injury
}

\author{
Kenichiro Nakamura ${ }^{a}$ b Yoshiyuki Tsukamoto $^{a}$ Naoki Hijiya ${ }^{a}$ Yasunori Higuchi ${ }^{d}$ \\ Shinji Yanoc Shigeo Yokoyamac Toshihide Kumamotob Masatsugu Moriyama ${ }^{\mathrm{a}}$ \\ Departments of a Molecular Pathology, ${ }^{\mathrm{b}}$ Neurology and Neuromuscular Disorders Science, and \\ 'Diagnostic Pathology, Faculty of Medicine, Oita University, and ${ }^{\mathrm{d}}$ Research Center for Applied Medical \\ Engineering, Oita University, Yufu City, Japan
}

\section{Key Words}

Uridine diphospho- $\mathrm{N}$-acetylglucosamine 2-epimerase/

$\mathrm{N}$-acetylmannosamine kinase $\cdot$ Muscle $\cdot$ Regeneration

\begin{abstract}
Objective: The aim of the present study was to clarify the expression of uridine diphospho- $\mathrm{N}$-acetylglucosamine 2-epimerase/N-acetylmannosamine kinase (GNE) protein and mRNA in damaged or regenerating myofibers. Methods: We investigated the muscle expression pattern of GNE protein by immunohistochemistry using a murine model involving intramuscular injection of cardiotoxin (CTX), and the expression level of GNE mRNA by quantitative real-time polymerase chain reaction analysis of damaged or regenerating myofibers that had been collected directly from tissue sections using laser-capture microdissection. Results: The expression of GNE protein was increased in severely damaged myofibers as well as in regenerating myofibers with central nuclei, both of which also showed an increase in the expression of GNE mRNA. In regenerating myofibers, immunoreactivity for GNE protein in nuclei relative to that in the cytoplasm was higher at 7 days than at 4 days after CTX injection. Conclusion: Our findings suggest that GNE expression is induced when myofibers are damaged or regenerating, and that GNE plays a role in muscle regeneration.
\end{abstract}

Copyright $\odot 2010$ S. Karger AG, Basel

\section{Introduction}

The GNE gene encodes uridine diphospho-N-acetylglucosamine 2-epimerase/ $\mathrm{N}$-acetylmannosamine kinase (GNE), a key enzyme in the sialic acid biosynthetic pathway [1-3]. Sialic acids are expressed as terminal carbohydrates on glycoconjugates of eukaryotic cells, and function in cellular interactions and signaling $[3,4]$. Under physiological conditions, GNE is expressed in various tissues, including muscle in mice as well as humans [5-7]. Deletion of the GNE gene in mice is embryonically lethal, suggesting that GNE plays an important role in development [8]. It is widely accepted that germ line mutation of the GNE gene is responsible for the pathogenesis of an autosomal recessive muscle disease with early adult onset, distal myopathy with rimmed vacuoles [9] and hereditary inclusion body myopathy [10]. Distal myopathy with rimmed vacuoles/hereditary inclusion body myopathy is characterized clinically by gradually progressive limb muscle weakness and atrophy, and pathologically by the presence of many cytoplasmic rimmed vacuoles, intranuclear tubulofilamentous inclusions and myofiber atrophy with little evidence of necrosis or regeneration [11-14]. It has recently been reported that the expression and subcellular distribution of GNE protein in muscle from patients with hereditary inclusion body myopathy are similar to those in normal muscle [15]. However, it is

\section{KARGER \\ Fax +41613061234 E-Mail karger@karger.ch} www.karger.com

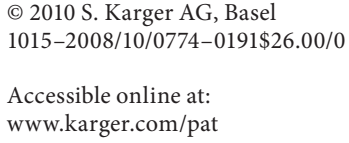

Masatsugu Moriyama, $\mathrm{MD}, \mathrm{PhD}$

Department of Molecular Pathology

Faculty of Medicine, Oita University

Yufu City, Oita 879-5593 (Japan)

Tel. +8197586 5690, Fax +81 97586 5699, E-Mail mmoriyam@med.oita-u.ac.jp 
unknown whether the expression pattern of GNE in vivo changes in the regeneration process after muscle injury. In the present study, we investigated the expression pattern of GNE during the regeneration process after muscle injury induced by injection of cardiotoxin (CTX), and found that GNE is induced in both severely damaged myofibers and regenerating myofibers after muscle injury.

\section{Materials and Methods}

\section{Animals and CTX Injection}

C57BL/6 male mice (Kyudo, Saga, Japan) aged 7-12 weeks were used in this study. All experiments were performed in accordance with the guidelines for animal care and use at Oita University (Permission No. H008001). After anesthesia, $50 \mu \mathrm{l}$ of 10 $\mu \mathrm{M}$ CTX (Latoxan, Rosans, France) was injected into the right gastrocnemius muscle. At 1, 2, 4, and 7 days after CTX injection, mice were sacrificed and the gastrocnemius muscles were resected. Muscles were fixed with $4 \%$ paraformaldehyde and embedded in paraffin, or immediately frozen in liquid nitrogen-cooled isopentane and stored at $-80^{\circ} \mathrm{C}$. Noninjected gastrocnemius muscles were used as control.

\section{Generation of GNE Antibodies}

Total RNA was extracted from a human monocytic leukemia cell line (THP-1). The cDNA fragment encoding the full length of the GNE protein was synthesized and amplified by reverse-transcription polymerase chain reaction (RT-PCR) with the forward primer $5^{\prime}$-ACCTCTCAAAACGAAACAAG-3' and the reverse primer 5'-AAGAAACGGTCATCCTAAAG-3'. The PCR product was subcloned into pGEM-T easy vector (Promega, Madison, Wisc., USA) and sequenced (GNE-pGEM-T easy). Although plurally cloned cDNAs have the same mutation (A to G) at codon 309, we used this clone in the following experiment, since this mutation may not affect the amino acid sequence.

Next, in order to generate recombinant GNE protein fused to glutathione-S-transferase (GST), cDNAs encoding the N-terminal region (1-380 amino acids) of GNE (GNE-E) and the C-terminal region (410-722 amino acids) of GNE (GNE-K) were amplified by PCR with the following primers: GNE-E forward, 5' CGCGGATCCATGGAGAAGAACGGGAAT; GNE-E reverse, 5'-CCGCTCGAGTCATGAAGGTCGATGGATTTG; GNE-K forward, 5'-CGCGGATCCTTGGCTGTTGATCTCGGG; GNE$\mathrm{K}$ reverse, 5'-CCGCTCGAGCTAGTGGATCCTGCGGGT and GNE-pGEM-T easy as the template. Following sequencing, the cDNAs were subcloned into the BamHI and XhoI sites of the pGEX4T-1 vector plasmid (Amersham Biosciences, Piscataway, N.J., USA), respectively. The construct of the full-length GNE fused to GST (GST-GNE) was done by cloning from GNE-pGEMT easy to pGEX4T-1 at the EcoRI site. GST-GNE-E, GST-GNE-K and GST-GNE were synthesized and purified as described previously [16].

The purified GST-GNE-E and GST-GNE-K proteins were used to immunize rabbits to raise anti-GNE polyclonal antibodies. The antibodies were affinity purified as described previously [17]. The purified antibodies, designated ' $\alpha$-GNE-E Ab' and ' $\alpha$ -
GNE-K Ab' and directed at the GST-GNE-E and GST-GNE-K proteins, respectively, were used in the following experiments.

The additional primary and secondary antibodies listed in online supplementary table 1 (for all supplementary material, see www.karger.com/doi/10.1159/000292652) were obtained commercially and used according to the manufacturer's instructions.

Histological Analysis

The cross-sectional area (CSA) of the regenerating myofibers and the gastrocnemius muscle were measured using NIH ImageJ software. The CSA of the regenerating myofibers $\left(\mu \mathrm{m}^{2}\right)$ and the number of damaged or regenerating myofibers per unit CSA $\left(\mathrm{mm}^{2}\right)$ were expressed as means $\pm \mathrm{SD}$. Differences between variances were tested by the Mann-Whitney U test as a nonparametric method.

\section{Immunohistochemistry and Double-Labeled}

Immunofluorescence

Immunohistochemistry and double-labeled immunofluorescence were performed as described previously [18]. Briefly, paraffin-embedded tissue sections were deparaffinized and rehydrated using standard protocols. Tissue sections from frozen specimens were fixed in $4 \%$ paraformaldehyde for $20 \mathrm{~min}$ at room temperature (RT). For antigen retrieval, sections were immersed in $10 \mathrm{mM}$ sodium citrate buffer, $\mathrm{pH} 6.0$ (Iatron, Tokyo, Japan), autoclaved at $120^{\circ} \mathrm{C}$ for $10 \mathrm{~min}$ and cooled to RT. They were treated with $3 \%$ $\mathrm{H}_{2} \mathrm{O}_{2}$ for 5 min at RT to inactivate endogenous peroxidase activity for avidin-biotin complex methods. After blocking with $10 \%$ goat serum (Nichirei, Tokyo, Japan) for $30 \mathrm{~min}$ at RT, immunohistochemistry with rabbit polyclonal $\alpha$-GNE-K Ab was performed by incubating the tissue sections overnight at $4^{\circ} \mathrm{C}$ with the first antibody at concentrations of $4 \mu \mathrm{g} / \mathrm{ml}$ in diluting solution (Dako, Copenhagen, Denmark).

For avidin-biotin complex methods, the sections were then washed with $1 \times$ phosphate-buffered saline (PBS) and incubated for 30 min with biotinylated goat $\alpha$-rabbit IgG antibody (Nichirei). After being washed, they were incubated with a solution of avidin-conjugated horseradish peroxidase (Vectastain Elite ABC kit; Vector Laboratories Inc., Burlingame, Calif., USA) for $15 \mathrm{~min}$, according to the manufacturer's recommendations, and then washed. Peroxidase activity was detected with $\mathrm{H}_{2} \mathrm{O}_{2}$ /diaminobenzidine substrate solution, and the sections were counterstained with hematoxylin before dehydration and mounting.

For double-labeled immunofluorescence, sections incubated with a mixture of appropriately diluted first antibodies were then washed with $1 \times$ PBS and incubated with a mixture of appropriately diluted secondary antibodies or propidium iodide (Sigma, Tokyo, Japan) for $2 \mathrm{~h}$ at RT. They were then washed and mounted. The mounted sections were observed using the LSM5 Pascal confocal laser scanning microscope (Carl Zeiss, Oberkochen, Germany).

For antigen competition experiments, prior to immunostaining $\alpha$-GNE-K Ab was preabsorbed to more than a 700 -fold weight excess of GST-GNE-K.

\section{Western Blotting}

Fresh-frozen samples were ground to a fine powder with liquid nitrogen. Total proteins were extracted as described previously [19]. Western blotting analysis was performed as described previously [20]. 
Extraction of Total RNA from Laser-Capture Microdissected Myofiber Samples

Tissue sections $10 \mu \mathrm{m}$ thick were cut from frozen blocks with a cryostat Leica CM1850 (Leica Microsystems, Wetzlar, Germany), and then stained with cresyl violet (Ambion). Damaged, regenerating or control myofibers were collected from the tissue sections using the Arcturus ${ }^{\mathrm{XT}}$ microdissection system (Arcturus Engineering Inc., Mountain View, Calif., USA). Control myofibers (with a normal appearance) were collected from the same tissue sections from which damaged or regenerating myofibers had also been collected. The collected myofibers were dissolved immediately in Isogen reagent (Nippon Gene, Tokyo, Japan), and total RNA was isolated as described by the manufacturer. Reverse transcription was carried out using a Transcriptor first-strand cDNA synthesis kit (Roche, Mannheim, Germany) with random hexamer primers, in accordance with the manufacturer's instructions.

\section{Quantitative PCR for GNE}

Quantitative real time PCR (qRT-PCR) was performed using LightCycler FastStart DNA Master SYBR Green I (Roche, Mannheim, Germany) and analyzed in a LightCycler 2.0 (Roche) with LightCycler Software version 4.0 (Roche), according to the manufacturer's instructions. Primers for mouse GNE (MA065432) (forward, 5'-ACGTGAAGGCCCAGAGCATC; reverse, 5'-TGACTGGCCAGGACTCCAGA) and mouse GAPDH (MA050371) (forward, 5'-TGTGTCCGTCGTGGATCTGA; reverse, 5'-TTGCTGTTGAAGTCGCAGGAG) were designed by and purchased from Takarabio (Shiga, Japan). Dilutions of cDNA synthesized from noninjected murine gastrocnemius muscles were used as standards. qRT-PCR was carried out using the following cycle parameters: 1 cycle of $10 \mathrm{~min}$ at $95^{\circ} \mathrm{C}$, followed by 40 cycles of $10 \mathrm{~s}$ at $95^{\circ} \mathrm{C}, 10 \mathrm{~s}$ at $60^{\circ} \mathrm{C}$, and $20 \mathrm{~s}$ at $72^{\circ} \mathrm{C}$. Melting curve analysis was performed to confirm the correct PCR product. GAPDH was used as a control and relative expression levels were obtained by relative quantification analysis. PCR amplification was duplicated in independent experiments.

\section{Immunoelectron Microscopy}

The gastrocnemius muscles at 7 days after CTX injection were fixed in $4 \%$ paraformaldehyde overnight, followed by routine embedding in paraffin. Tissue sections stained with hematoxylin and eosin (HE) were then prepared and observed by light microscopy, and we selectively obtained tissues that were rich in regenerating myofibers. The selected tissues were cut into small pieces with a knife, deparaffinized in xylene, rehydrated in ethanol, and fixed overnight at $4{ }^{\circ} \mathrm{C}$ in a mixture of $2 \%$ paraformaldehyde, $0.5 \%$ glutaraldehyde, $0.2 \mathrm{mM} \mathrm{CaCl}_{2}$, and $0.1 \mathrm{mM} \mathrm{MgCl}_{2}$ in $0.1 \mathrm{M}$ sodium cacodylate buffer, $\mathrm{pH} 7.4$, which was supplemented with tannic acid at a final concentration of $0.1 \%$, just before use. The pieces were dehydrated in an ascending graded ethanol series and infiltrated with LR White resin (London Resin Company, UK), which was polymerized by heating at $55^{\circ} \mathrm{C}$ for $24 \mathrm{~h}$. LR White-embedded blocks for immunoelectron microscopy using $\alpha$-GNE-K Ab were prepared as described previously $[18,21]$. Briefly, ultrathin sections were cut with an ultramicrotome (Leica Instruments, Nussloch, Germany) at a thickness of $90 \mathrm{~nm}$ and mounted on nickel grids that had been coated with carbon in a vacuum evaporator. For immunostaining, nickel grids bearing sections were immersed in an alkaline solution (Target Retrieval Solution, $\mathrm{pH}$ 10;
Dako, Carpinteria, Calif., USA), heated at $95^{\circ} \mathrm{C}$ for $30 \mathrm{~min}$, and then cooled to RT. After blocking, the sections were incubated with $\alpha$-GNE-K Ab $(5 \mu \mathrm{g} / \mathrm{ml})$ diluted with $1 \times$ Tris-buffered saline containing $0.1 \%(\mathrm{w} / \mathrm{v})$ bovine serum albumin at $4^{\circ} \mathrm{C}$ for $18 \mathrm{~h}$. After washing, the sections were incubated with the appropriately diluted 15-nm gold particle-conjugated secondary antibody at RT for $30 \mathrm{~min}$. After washing, the sections were counterstained with uranyl acetate and lead citrate, and observed with a transmission electron microscope (JEM 1230, Jeol Ltd., Tokyo, Japan). Preparations of normal quadriceps muscle were made in a similar way. Negative controls were prepared by omitting the first antibody.

\section{Results}

\section{Characterization of Anti-GNE Antibodies}

We generated two affinity-purified anti-GNE antibodies by immunizing rabbits with the recombinant GNE protein fused to GST containing the epimerase domain corresponding to amino acids 1-380 (GNE-E), or with that containing the kinase domain corresponding to amino acids 410-722 (GNE-K), as described in Materials and Methods (online suppl. fig. 1A). On Western blots, while $\alpha$-GNE-E Ab and $\alpha$-GNE-K Ab were specifically immunoreactive with GNE-E and GNE-K, respectively (online suppl. fig. 1B), both of the antibodies were immunoreactive with purified full-length GNE proteins fused to GST (online suppl. fig. 1B) as well as with full-length GNE proteins fused to GFP exogenously expressed in HEK293 cells (online suppl. fig. 1C). As shown in online supplementary figure 1D, Western blotting analysis of extracts from the murine muscle tissue showed that both $\alpha-$ GNE-E Ab and $\alpha$-GNE-K Ab detected a band at about $79 \mathrm{kDa}$ corresponding to GNE [7]. Immunohistochemical analysis of the normal murine muscle showed that positive immunoreactivity for $\alpha$ GNE-K Ab was detected mainly in the cytoplasm and occasionally in the nuclei of myofibers (fig. 1A). Nonspecific immunoreactivity was unlikely because these positive immunoreactivities were found to be significantly decreased in the antigen competition experiments (fig. 1B). Double-labeled immunohistochemistry with $\alpha$ GNE-K Ab showed that the expression of GNE protein overlapped with that of $\alpha$-actinin (online suppl. fig. $1 \mathrm{E}-$ $\mathrm{G})$, in accord with the findings of a previous study [22]. Immunoelectron microscopy analysis of normal murine muscle with $\alpha$-GNE-K Ab showed that immunolabeled gold particles were attached to the sarcomeric Z-lines and I-bands (fig. 2A). 
Fig. 1. Immunohistochemical analysis of GNE in CTX-injected murine gastrocnemius muscles. A-F Paraffin-embedded tissue sections from noninjected murine gastrocnemius muscles $(\mathbf{A}, \mathbf{B})$, and at 1 day $(\mathbf{C}$, D) and 7 days $(\mathbf{E}, \mathbf{F})$ after CTX injection were immunostained with $\alpha$-GNE-K Ab. Positive immunoreactivity $(\mathbf{A}, \mathbf{C}, \mathbf{E})$ is significantly decreased in competition experiments (B, D, F). G-I Tissue sections from murine gastrocnemius muscles at 2 days after CTX injection were analyzed by double-labeled immunostaining analysis with $\alpha$-GNE-K Ab (green) together with $\alpha$-desmin antibody (red). Immunoreactivity for desmin is positive in viable myofibers and negative in damaged myofibers. Results were similar in at least 3 independent experiments. Scale bars: $50 \mu \mathrm{m}(\mathbf{A}, \mathbf{B}, \mathbf{E}, \mathbf{F})$, $100 \mu \mathrm{m}(\mathbf{C}, \mathbf{D})$ and $20 \mu \mathrm{m}(\mathbf{I})$.
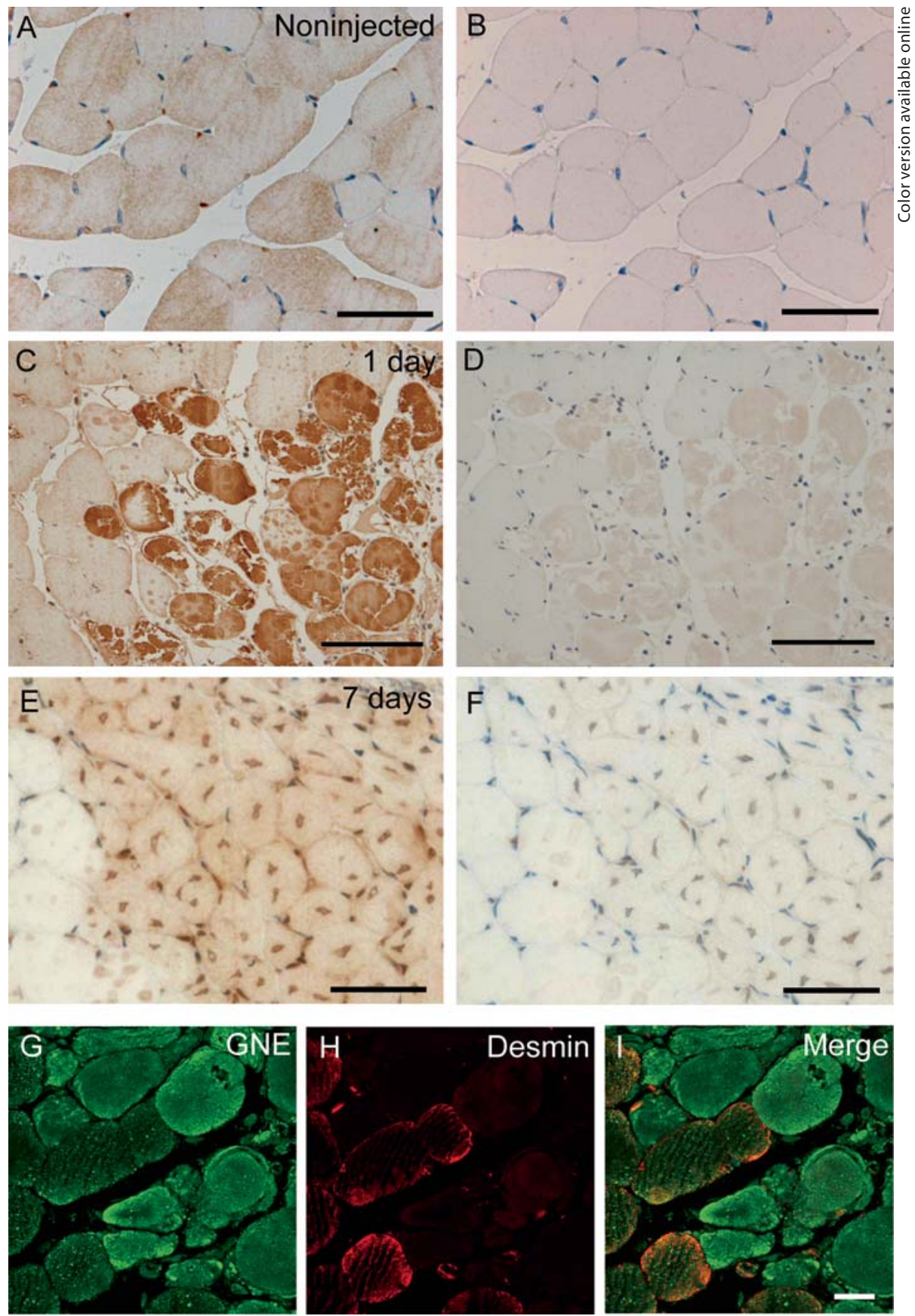

Expression of GNE Protein Is Increased in Damaged or Regenerating Myofibers of CTX-Injected Mice

To determine whether the expression pattern of GNE is changed during the process of regeneration after muscle injury, we investigated the expression pattern of GNE in the gastrocnemius muscle after intramuscular injection of CTX. In accordance with previous reports [23, 24], histological analysis showed that many destroyed or damaged myofibers were present at 1 and 2 days after CTX injection (online suppl. fig. 2A, B). At 4 days after CTX injection, while the number of damaged myofibers had decreased $(38.1 \pm 5.8,39.1 \pm 7.4$, and $1.1 \pm 1.2$ myofibers per square millimeter CSA at 1,2, and 4 days after CTX injection, respectively, $\mathrm{n}=3, \mathrm{p}=0.04$ ), basophilic regenerating myofibers with central nuclei were present together with small mononuclear cells, probably includ- 

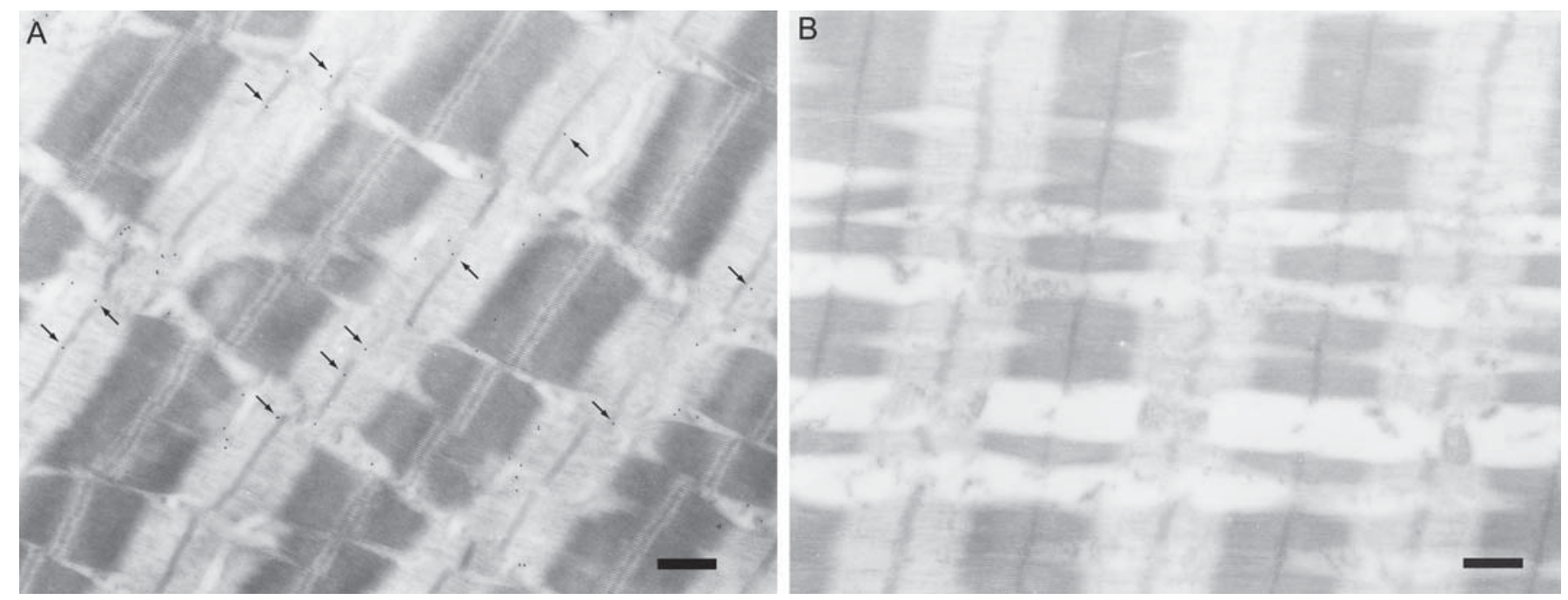

Fig. 2. Immunoelectron microscopy analysis of normal murine quadriceps muscle using $\alpha-\mathrm{GNE}-\mathrm{K} A \mathrm{Ab}(\mathbf{A})$, and a negative control omitting the first antibody (B). Arrows indicate GNE protein localized to the Z-line. Scale bar: $0.5 \mu \mathrm{m}$.

ing activated satellite cells, fibroblasts and macrophages (online suppl. fig. 2C). At 7 days after CTX injection, the number of regenerating myofibers with central nuclei had increased $(79.3 \pm 31.1$ and $188.5 \pm 69.6$ myofibers per square millimeter CSA at 4 and 7 days after CTX injection, respectively, $\mathrm{n}=4, \mathrm{p}=0.02$ ) (online suppl. fig. 2D).

Immunohistochemical analysis demonstrated strongly positive immunoreactivity with $\alpha$-GNE-K Ab in myofibers corresponding to those that had been found to be damaged on histological analysis 1 and 2 days after CTX injection (fig. 1C). This positive immunoreactivity was significantly decreased by antigen competition (fig. 1D). Double-labeled immunohistochemistry demonstrated that desmin was downregulated in myofibers that were strongly GNE positive (fig. 1G-I), suggesting that these myofibers were severely damaged [25-29]. Furthermore, Western blotting analysis revealed that the expression level of GNE protein was significantly increased at 1 and 2 days after CTX injection (online suppl. fig. 3).

Positive immunoreactivity for $\alpha$-GNE-K Ab was also detected in both the cytoplasm and the central nuclei of regenerating myofibers at 7 days after CTX injection, and the immunoreactivity in regenerating myofibers was stronger than that in nondamaged mature myofibers with peripheral nuclei (fig. 1E). This immunoreactivity was significantly decreased by antigen competition (fig. 1F). We found that the immunoreactivity for GNE protein in the nuclei relative to that in the cytoplasm of regenerating myofibers was higher at 7 days than at 4 days after CTX injection (fig. 3A-F). The CSA of regenerating myofibers was significantly increased at 7 days (492.1 \pm $193.4 \mu \mathrm{m}^{2}, 276$ myofibers) compared with that at 4 days $\left(188.0 \pm 72.5 \mu \mathrm{m}^{2}, 263\right.$ myofibers) after CTX injection $(\mathrm{p}<0.0001)$. Double-labeled immunohistochemistry showed that some small mononuclear cells expressing GNE coexpressed myogenin (fig. 3G-L), suggesting a population of activated satellite cells.

Immunoelectron microscopy analysis using $\alpha$-GNE$\mathrm{K} \mathrm{Ab}$ showed immunolabeled gold particles attached to the central nucleus; immunolabeled gold particles were also present in the cytoplasm of regenerating myofibers at 7 days after CTX injection (fig. 4A, B). Positive signals were not distributed diffusely in the nucleus but were detectable in the peripheral heterochromatin (fig. 4B) as well as internal electron-dense regions (fig. 4B). Furthermore, positive signals were also detectable at the nucleolus (fig. 4B).

\section{Induction of GNE in Damaged or Regenerating Myofibers Confirmed by $q R T-P C R$}

To confirm the induction of GNE in damaged or regenerating myofibers, we selectively collected damaged or regenerating myofibers from sections of CTX-injured muscle tissue using laser-capture microdissection, and then measured the expression level of GNE mRNA by qRT-PCR analysis. Consistent with the immunohistochemical data (fig. 1C), the expression level of GNE mRNA in damaged myofibers was evidently increased in 

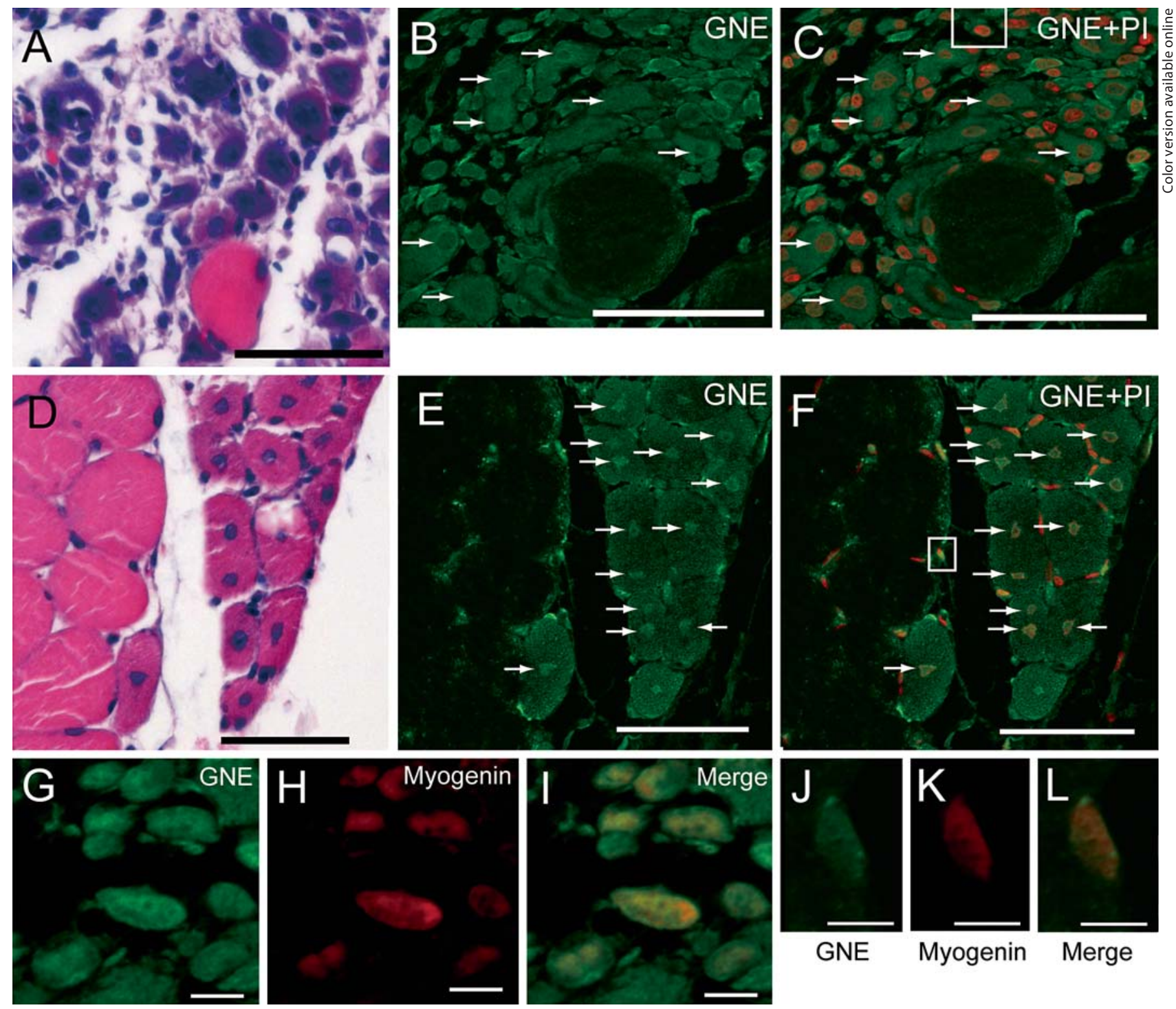

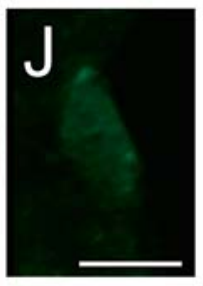

GNE

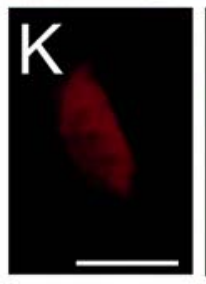

Myogenin

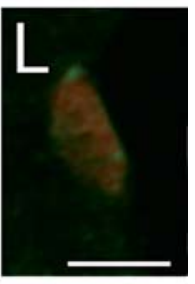

Merge
Fig. 3. Double-labeled immunofluorescence of GNE in regenerating muscle. Tissue sections from murine gastrocnemius muscles at 4 days (A-C and $\mathbf{G}-\mathbf{I})$ and 7 days (D-F, $\mathbf{J}-\mathbf{L})$ after CTX injection were stained by $\mathrm{HE}(\mathbf{A}, \mathbf{D})$, immunostaining with $\alpha-\mathrm{GNE}-\mathrm{K} \mathrm{Ab}$ (green) and nuclear staining (red) with propidium iodide (PI) after the sections had been incubated with ribonuclease (Sigma, To- kyo, Japan) (B, C, E, F), and double-labeled immunostaining with $\alpha$-GNE-K Ab (green) together with $\alpha$-myogenin antibody (red) (G-L). G-I and $\mathbf{J}-\mathbf{L}$ correspond to the boxed areas in $\mathbf{C}$ and $\mathbf{F}$, respectively. Arrows indicate central nuclei in regenerating myofibers. Scale bars: $50 \mu \mathrm{m}(\mathbf{A}-\mathbf{F})$ and $5 \mu \mathrm{m}(\mathbf{G}-\mathbf{L})$. comparison with that in nondamaged myofibers (fig. 5A). Furthermore, the expression level of GNE mRNA in regenerating myofibers was also increased in comparison with that in control myofibers (fig. 5B). Thus, we concluded that GNE is induced in both damaged and regenerating myofibers.

\section{Discussion}

We found that the expression level of GNE was increased in damaged and regenerating myofibers. GNE was inducible not only by intramuscular injection of CTX but also by touching the muscle with dry ice (data not 


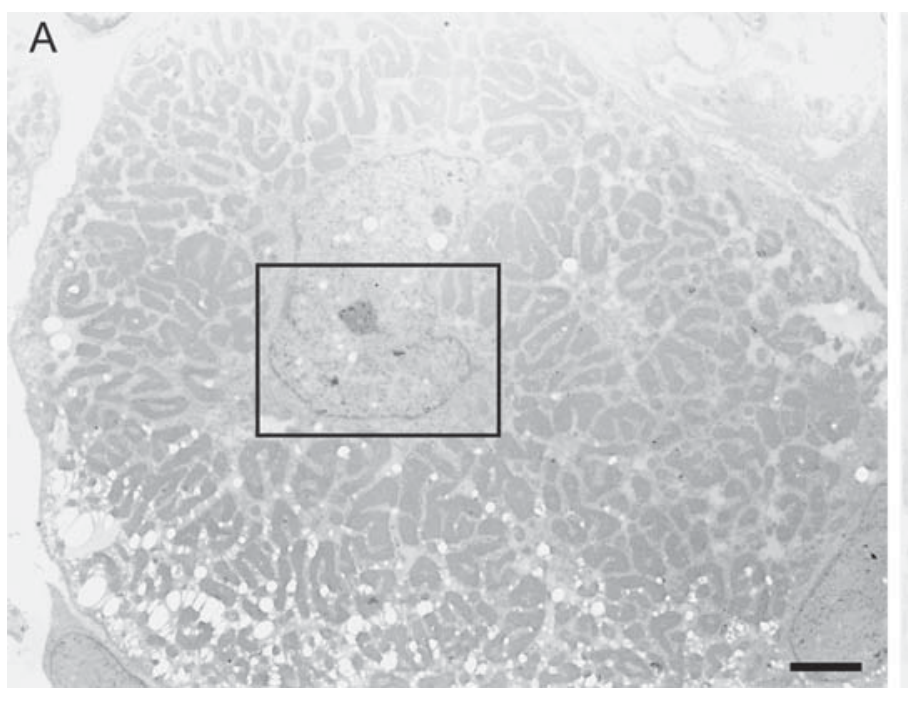

Fig. 4. Immunoelectron microscopy analysis of GNE in regenerating myofibers at 7 days after CTX injection. A A representative regenerating myofiber with a central nucleus. B High-power view

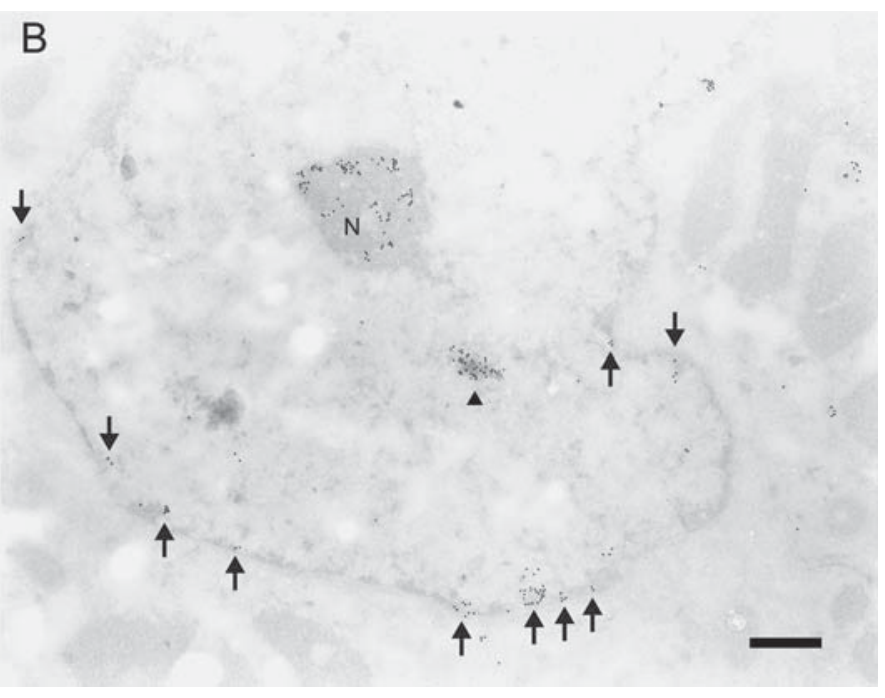

of the boxed area in A. The arrowhead indicates the internal electron-dense region. Arrows indicate the peripheral heterochromatin. $\mathrm{N}=$ Nucleolus. Scale bars: $2 \mu \mathrm{m}(\mathbf{A})$ and $0.5 \mu \mathrm{m}(\mathbf{B})$.
Fig. 5. Upregulation of GNE mRNA in damaged or regenerating myofibers. Damaged myofibers at 1 day and 2 days after CTX injection (A) and regenerating myofibers at 7 days after CTX injection (B) were selectively collected using laser-capture microdissection. The expression levels of GNE mRNA in damaged myo-fibers (A) and regenerating myofibers (B) were analyzed by qRT-PCR, and expressed as the fold change compared with the control (nondamaged myofibers). GAPDH mRNA was used as an internal control.

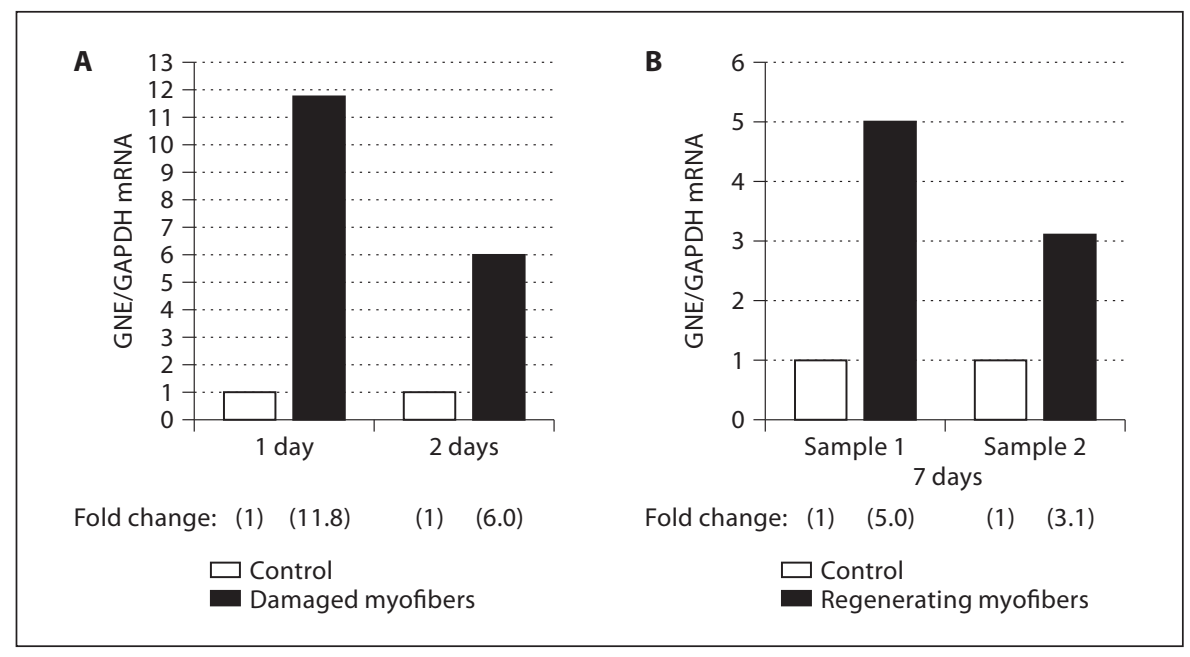

shown), suggesting that induction of GNE is not specific to CTX but rather can be induced by various kinds of muscle damage.

We showed that not only the expression level of GNE mRNA but also that of GNE protein was increased in damaged myofibers showing downregulation of desmin. Proteolysis is usually activated in damaged myofibers $[28,29]$. Nevertheless, upregulation of some proteins, such as calcium-dependent proteinase, calpain, and its endogenous inhibitor calpastatin [30,31], proteasomes [32], Bcl-2 and Bax [33], has been reported in necrotic myofibers. Myofibrillar protein degradation in CTX-induced myonecrosis, for example, is compatible with the 'two-step' mechanism; the first stage is associated with cytosolic muscle proteinases, mainly calpains, responsible for limited proteolysis of a few key structural proteins such as desmin; the second stage predominantly involves leukocyte and macrophage proteinases, which cause 
widespread degradation of the most abundant myofibrillar components $[28,29]$. These results suggest that proteolysis in damaged myofibers is not uniform. We speculate that GNE protein in damaged myofibers is stable, at least in comparison with desmin. Future studies of the mechanism responsible for degradation of GNE protein will help answer this question.

Neural cell adhesion molecules (NCAM) are reported to be polysialylated in regenerating myofibers in vivo and in myotubes in vitro $[34,35]$, suggesting that sialic acid has a role in muscle regeneration. It has also been reported that a polysialylated form of NCAM (PSA-NCAM) is expressed in regenerating myofibers as well as activated satellite cells after muscle injury induced by sodium dihydrogen phosphate [36]. In this model, the regeneration process is similar to that induced by CTX. PSA-NCAM is reported to be associated with cell rearrangement and migration during development [37]. However, the role of PSA-NCAM in muscle regeneration remains to be elucidated. It has recently been reported that polysialylation of NCAM is reduced in the brain of heterozygous GNE-deficient mice [38] as well as in GNE-deficient mouse embryonic stem cells [8]. Therefore, we speculate that GNE is induced and contributes to the regulation of sialic acid biosynthesis in regenerating myofibers.

In addition, we found that GNE protein was markedly localized in nuclei of regenerating myofibers at 7 days, as compared with that at 4 days after CTX injection, suggesting that the function of GNE alters during the regen- eration process. The mechanism responsible for the nuclear localization of GNE is still unclear, although its translocation from the cytoplasm to the nucleus may be dependent upon some intracellular signaling process. Alternatively, the relative stability of the protein may differ between the nucleus and the cytoplasm in regenerating myofibers. It has recently been reported that promyelocytic leukemia zinc finger protein (PLZF) binds to GNE [39]. PLZF is a sequence-specific DNA-binding transcriptional repressor associated with corepressor molecules such as N-CoR, Sin3A, SMRT and HDAC1 [40-43]. It has already been reported that the cytoplasmic enzyme glyceraldehyde-3-phosphate dehydrogenase translocates to the nucleus in response to oxidative stress and associates with the regulator of transcription factors, APE1 [44]. Therefore, we speculate that GNE functions as a transcriptional regulator in regenerating myofibers via binding to such corepressor complexes.

\section{Acknowledgments}

This research was supported in part by the Ministry of Education, Science, Sports and Culture, Grant-in-Aid for Scientific Research (C), No. 15590898 and No. 18590952 and by Research on intractable diseases, Health and Labour Sciences Research Grants from the Ministry of Health, Labour and Welfare, Japan. We are grateful to Tsuyoshi Iwao, Mariko Ono and Kaori Hirano for excellent technical assistance.

\section{References}

$\rightarrow$ Hinderlich S, Stäsche R, Zeitler R, Reutter W: A bifunctional enzyme catalyzes the first two steps in $\mathrm{N}$-acetylneuraminic acid biosynthesis of rat liver. Purification and characterization of UDP-N-acetylglucosamine 2-epimerase/ $\mathrm{N}$-acetylmannosamine kinase. J Biol Chem 1997;272:24313-24318.

-2 Stäsche R, Hinderlich S, Weise C, et al: A bifunctional enzyme catalyzes the first two steps in $\mathrm{N}$-acetylneuraminic acid biosynthesis of rat liver. Molecular cloning and functional expression of UDP-N-acetylglucosamine 2-epimerase/ $\mathrm{N}$-acetylmannosamine kinase. J Biol Chem 1997;272:24319-24324.

- 3 Keppler OT, Hinderlich S, Langner J, Schwartz-Albiez R, Reutter W, Pawlita M: UDP-GlcNAc 2-epimerase: a regulator of cell surface sialylation. Science 1999;284: 1372-1376.

4 Tanner ME: The enzymes of sialic acid biosynthesis. Bioorg Chem 2005;33:216-228.
5 Horstkorte R, Nöhring S, Wieschens N, et al: Tissue expression and amino acid sequence of murine UDP-N-acetylglucosamine 2epimerase/N-acetylmannosamine kinase. Eur J Biochem 1999;260:923-927.

-6 Lucka L, Krause M, Danker K, Reutter W, Horstkorte R: Primary structure and expression analysis of human UDP-N-acetylglucosamine 2-epimerase/ $\mathrm{N}$-acetylmannosamine kinase, the bifunctional enzyme in neuraminic acid biosynthesis. FEBS Lett 1999;454:341-344.

7 Krause S, Hinderlich S, Amsili S, et al: Localization of UDP-GlcNAc 2-epimerase/ ManAc kinase (GNE) in the Golgi complex and the nucleus of mammalian cells. Exp Cell Res 2005;304:365-379.

8 Schwarzkopf M, Knobeloch K, Rohde E, Hinderlich S, Wiechens N, Lucka L, Horak I, Reutter W, Horstkorte R: Sialylation is essential for early development in mice. Proc Natl Acad Sci USA 2002;99:5267-5270.
-9 Nishino I, Noguchi S, Murayama K, et al: Distal myopathy with rimmed vacuoles is allelic to hereditary inclusion body myopathy. Neurology 2002;59:1689-1693.

10 Eisenberg I, Avidan N, Potikha T, et al: The UDP-N-acetylglucosamine 2-epimerase/Nacetylmannosamine kinase gene is mutated in recessive hereditary inclusion body myopathy. Nat Genet 2001;29:83-87.

11 Nonaka I, Sunohara N, Ishiura S, Satoyoshi E: Familial distal myopathy with rimmed vacuole and lamellar (myeloid) body formation. J Neurol Sci 1981;51:141-155.

12 Kumamoto T, Fukuhara N, Nagashima M, Kanda T, Wakabayashi M: Distal myopathy: histochemical and ultrastructural studies. Arch Neurol 1982;39:367-371.

13 Argov Z, Yarom R: 'Rimmed vacuole myopathy' sparing the quadriceps: a unique disorder in Iranian Jews. J Neurol Sci 1984;64: 33-43. 
14 Nonaka I, Murakami N, Suzuki Y, Kawai M: Distal myopathy with rimmed vacuoles. Neuromuscul Disord 1998;8:333-337.

15 Krause S, Aleo A, Hinderlich S, et al: GNE protein expression and subcellular distribution are unaltered in HIBM. Neurology 2007; 69:655-659.

16 Onizuka T, Moriyama M, Yamauchi T, et al: BCL-6 Gene Product, a 92- to 98-kD nuclear phosphoprotein, is highly expressed in germinal center B cells and their neoplastic counterparts. Blood 1995;86:28-37.

17 Moriyama M, Tsukamoto Y, Fujiwara M, et al: Identification of a novel human ankyrinrepeated protein homologous to CARP. Biochem Biophys Res Commun 2001;285:715723 .

18 Tsukamoto Y, Hijiya N, Yano S, et al: Arpp/ Ankrd2, a member of the muscle ankyrin repreat proteins (MARPs), translocates from the I-band to the nucleus after muscle injury. Histochem Cell Biol 2008;129:55-64.

19 Nakada C, Tsukamoto Y, Oka A, et al: Cardiac-restricted ankyrin-repeated protein is differentially induced in Duchenne and congenital muscular dystrophy. Lab Invest 2003; 83:711-719.

20 Uchida T, Kanada R, Tsukamoto Y, et al: Immunohistochemical diagnosis of the cagAgene genotype of Helicobacter pylori with anti-East Asian CagA-specific antibody. Cancer Sci 2007;98:521-528.

-21 Yano S, Kashima K, Daa T, et al: An antigen retrieval method using an alkaline solution allows immunoelectron microscopic identification of secretory granules in conventional epoxy-embedded tissue sections. J Histochem Cytochem 2003;51:199-204.

-22 Amsili S, Zer H, Hinderlich S, et al: UDP-Nacetylglucosamine 2-epimerase/ $\mathrm{N}$-acetylmannosamine kinase (GNE) binds to $\alpha$-actinin 1: novel pathways in skeletal muscle? PLoS One 2008;3:e2477.

23 Yan Z, Choi S, Liu X, et al: Highly coordinated gene regulation in mouse skeletal muscle regeneration. J Biol Chem 2003;278: 8826-8836.

24 Chargé SBP, Rudnicki MA: Cellular and molecular regeneration of muscle regeneration. Physiol Rev 2004;84:209-238.
25 Helliwell TR: Lectin binding and desmin staining during bupivacaine-induced necrosis and regeneration in rat skeletal muscle. J Pathol 1988;155:317-326.

26 Barash IA, Peters D, Fridén J, Lutz GJ, Lieber RL: Desmin cytoskeletal modifications after a bout of eccentric exercise in the rat. Am J Physiol Regul Integr Comp Physiol 2002; 283:R958-R963.

27 Lovering RM, DeDeyne PG: Contractile function, sarcolemma integrity, and the loss of dystrophin after skeletal muscle eccentric contraction-induced injury. Am J Physiol Cell Physiol 2004;286:C230-C238.

28 Gutiérrez JM, Ownby CL: Skeletal muscle degeneration induced by venom phospholipases $\mathrm{A}_{2}$ : insights into the mechanisms of local and systemic myotoxicity. Toxicon 2003; 42:915-931.

29 Harris JB, Vater R, Wilson M, Cullen MJ: Muscle fibre breakdown in venom-induced muscle degeneration. J Anat 2003;202:363372.

30 Kumamoto T, Ueyama H, Watanabe S, et al: Immunohistochemical study of calpain and its endogenous inhibitor in the skeletal muscle of muscular dystrophy. Acta Neuropathol 1995;89:399-403.

31 Sultan KR, Dittrich BT, Leisner E, Paul N, Pette D: Fiber type-specific expression of major proteolytic systems in fast- to slowtransforming rabbit muscle. Am J Physiol Cell Physiol 2001;280:239-247.

32 Kumamoto T, Fujimoto S, Ito T, Horinouchi H, Ueyama H, Tsuda T: Proteasome expression in the skeletal muscles of patients with muscular dystrophy. Acta Neuropathol 2000;100:595-602.

33 Olivé M, Ferrer I: Bcl-2 and Bax protein expression in human myopathies. J Neurol Sci 1999; 164:76-81.

34 Dubois C, Figarella-Branger D, Pastoret C, Rampini C, Karpati G, Rougon G: Expression of NCAM and its polysialylated isoforms during mdx mouse muscle regeneration and in vitro myogenesis. Neuromuscul Disord 1994;4:171-182.

35 Suzuki M, Angata K, Nakayama J, Fukuda M: Polysialic acid and mucin type o-glycans on the neural cell adhesion molecule differentially regulate myoblast fusion. J Biol Chem 2003;278:49459-49468.
36 Figarella-Branger D, Pellissier JF, Bianco N, Karpati G: Sequence of expression of MyoD1 and various cell surface and cytoskeletal proteins in regenerating mouse muscle fibers following treatment with sodium dihydrogen phosphate. J Neurol Sci 1999;170:151160.

37 Rutishauser U: Polysialic acid at the cell surface: biophysics in service of cell interactions and tissue plasticity. J Cell Biochem 1998;70: 304-312.

-38 Gagiannis D, Orthmann A, Danssmann I, Schwarzkopf M, Weidemann W, Horstkorte $\mathrm{R}$ : Reduced sialylation status in UDP-Nacetylglucosamine-2-epimerase/N-acetylmannosamine kinase (GNE)-deficient mice. Glycoconj J 2007;24:125-130.

-39 Weidemann W, Stelzl U, Lisewski U, et al: The collapsin response mediator protein 1 (CRMP-1) and the promyelocytic leukemia zinc finger protein (PLZF) bind to UDP-Nacetylglucosamine 2-epimerase/ $\mathrm{N}$-acetylmannosamine kinase (GNE), the key enzyme of sialic acid biosynthesis. FEBS Lett 2006;580:6649-6654.

40 Li JY, English MA, Ball HJ, Yeyati PL, Waxman S, Licht JD: Sequence-specific DNA binding and transcriptional regulation by the promyelocytic leukemia zinc finger protein. J Biol Chem 1997;272:22447-22455.

41 Grignani F, Matteis SD, Nervi C, et al: Fusion proteins of the retinotic acid receptor- $\alpha$ recruit histone deacetylase in promyelocytic leukemia. Nature 1998;391:815-818.

$\checkmark 42$ Lin RJ, Nagy L, Inoue S, Shao W, Miller WH Jr, Evans RM: Role of the histone deacetylase complex in acute promyelocytic leukemia. Nature 1998;391:811-814.

43 Melnick AM, Westendorf JJ, Polinger A, et al: The ETO protein disrupted in $\mathrm{t}(8 ; 21)$-associated acute myeloid leukemia is a corepressor for the promyelocytic leukemia zinc finger protein. Mol Cell Biol 2000;20:20752086.

44 Azam S, Jouvet N, Jilani A, et al: Human glyceraldehyde-3-phosphate dehydrogenase plays a direct role in reactivating oxidized forms of the DNA repair enzyme APE1. J Biol Chem 2008;283:30632-30641. 